\title{
Resource Allocation for OFDM-based Cognitive Radio Multicast Networks
}

\author{
Duy T. Ngo and Chintha Tellambura \\ Department of Electrical \& Computer Engineering, \\ University of Alberta, AB, CANADA T6G 2 V4. \\ Email: \{trongduy,chintha\}@ece.ualberta.ca
}

\author{
Ha H. Nguyen \\ Department of Electrical \& Computer Engineering, \\ University of Saskatchewan, SK, CANADA S7N 5A9. \\ Email: ha.nguyen@usask.ca
}

\begin{abstract}
In cognitive radio networks with the coexistence of primary and secondary users, the problem of how to optimally allocate available resources (e.g., bandwidth and power) to multicast groups of secondary users that use orthogonal frequency division multiplexing (OFDM) is important. Taking the maximization of the weighted sum rate of such groups as the design objective, we propose a practically optimal subcarrier and power allocation scheme under constraints on the tolerable interference thresholds at individual primary user's frequency bands. Specifically, the optimization problem is solved via the dual method, where subcarriers are assigned in a per-tone basis and power is distributed in a water-filling fashion. As the number of subcarriers becomes large, the dual-domain solution becomes the global optimum of the primal problem with the duality gap vanishing to zero. The proposed design is valid for both unicast and multicast transmissions, and its computational complexity is only linear in the number of subcarriers. The effects of adjacent subcarrier nulling technique, which is to reduce mutual interference between primary and secondary frequency bands, on the proposed scheme are also examined. The superiority of the dual approach is confirmed by numerical results.
\end{abstract}

\section{INTRODUCTION}

During the last few years, opportunistic secondary use of licensed radio spectrum has emerged as a promising idea to cope with the growing number of bandwidth-hungry wireless services. Cognitive radio [1] has been identified as an efficient technology to promote this idea by exploiting the existence of the spectrum portions unoccupied by the primary (licensed) users. In a spectrum sharing environment, the main challenges of a cognitive radio network are to guarantee a protection of the primary users from excessive interference induced by the secondary (unlicensed or cognitive) users as well as to provide the secondary users with some Quality-of-Service (QoS) requirements. However, interference due to the sharing of radio channel becomes an obstacle to meet those challenges.

Spectrum pooling is the opportunistic spectrum access approach that enables public access to already licensed frequency bands [2]. The basic idea is to merge spectral ranges from difference spectrum owners (e.g., military, trunked radios) into a common pool, from which the secondary users may temporarily rent spectral resources during idle periods of licensed users. In effect, the licensed system does not need to be changed while the secondary users still have opportunities to gain access into unused resources. Among many possible technologies for secondary users' transmission in spectrum- pooling radio systems, OFDM (orthogonal frequency division multiplexing) has already been widely recognized as a highly promising candidate, mainly due to its great flexibility in dynamically allocating the unused spectrum among secondary users as well as its ability to monitor the spectral activities of licensed users at no extra cost. The study in [3] also shows that employing OFDM modulation causes mutual interference between the primary and secondary users due to the nonorthogonality of respective transmitted signals.

Although opportunistic spectrum access would allow secondary users to identify and access available spectrum resource, one of the major concerns is how to efficiently utilize this resource since radio spectrum is valuable but scarce in wireless communications. The resource allocation problem for OFDM-based cognitive radio networks has been examined in [4], where an optimal scheme, derived via Lagrangian formulation, is proposed to maximize the downlink capacity of the cognitive users while guaranteeing the interference introduced to the primary user being below a specified threshold. The work in [5] extends the results of [4] to the multiuser scenarios, where discrete sum rate of secondary network is maximized constrained on the interference induced to the primary user bands and on the total transmit power. Considering networks in which multiple secondary links coexist with multiple primary links through OFDMA-based air-interface, reference [6] utilizes the dual framework [7] to provide centralized and distributed algorithms which aim at maximizing the total sum rate of secondary networks subject to interference temperature constraints specified at primary users' receivers. All the above mentioned studies, however, only consider unicast transmission. On the other hand, multicasting, which is an efficient means of transmitting the same content to multiple receivers while minimizing the network resource usage [8], is certainly an attractive transmission technique for secondary networks who only have a limited access to the available spectrum.

In this paper, we design a practically optimal joint subcarrier assignment and power allocation scheme to maximize the weighted sum rate of all secondary users of an OFDMbased cognitive radio multicast network, while satisfying the tolerable interference level induced to the primary users. By utilizing the framework in [7], the original difficult nonconvex optimization problem is solved effectively in the dual domain with linear complexity in the number of subcarriers 
and with virtually zero duality gap as the number of subcarriers becomes large. Specifically, subcarriers are assigned in a pertone basis whereas power is allocated in a water-filling fashion.

It is worth pointing out that, although the main idea of the algorithms in [6] is the closest in spirit to the scheme proposed in this work as both studies are inspired by the results of [7], [9], the two are distinct in the following key aspects. First, while the model in [6] is likely to be applicable for ad-hoc unicast networks, the formulation investigated here is valid for more general scenarios including both unicast and multicast transmissions from BS (base station) to secondary users in cellular network context. Hence, the early results cannot be directly applied, and also the realization of distributed algorithm is, at best, impractical in multicast settings. Second, the primary users in [6] are restricted to employing OFDM whereas this is clearly not a requirement in this work. Our model is thus compatible with the spectrum pooling approach [2] wherein primary users have all the flexibility to deploy any desired transmission technique. Third, the mutual interference between secondary and primary users, which is an important factor, is explicitly quantified, and the effects of adjacent subcarrier nulling [3] on the proposed design are also analyzed in this paper.

\section{System Model and Problem Formulation}

Consider a one-cell wireless system, in which one BS transmits (not necessarily OFDM) signals to $N$ primary users, each of which occupies a determined frequency band $B_{P}^{n}(n=$ $1, \cdots, N)$ in the available spectrum. As the primary users do not utilize the entire available spectrum, the BS is permitted to employ OFDM and transmit $G$ downlink traffic flows, each to one distinct multicast group consisting of secondary users, over $K$ subcarriers in the unused frequency bands. Assume that each secondary user receives one traffic flow at a time, hence it belongs to only one multicast group. Let $M_{g}$ and $\left|M_{g}\right|(g=1, \cdots, G)$ denote the user set of group $g$ and its cardinality, respectively. The $g$-th group is unicast if $\left|M_{g}\right|=1$, whereas it is multicast if $\left|M_{g}\right|>1$. Clearly, all the secondary users belong to the set $M=\bigcup_{g=1}^{G} M_{g}$, and $|M|=\sum_{g=1}^{G}\left|M_{g}\right|$ is the total number of secondary users in the system. Let $B$ denote the total bandwidth sensed by the secondary users for possible transmission, and also assume that each subchannel has an equal bandwidth of $B_{s}=B / K$. It is further assumed that the spectrum available for transmission to multicast groups is located adjacent to that for transmission to primary users. The system setup is depicted in Fig. 1, and the distribution of the available spectrum is shown in Fig. 2.

Since the BS transmits (possibly non-orthogonal) signals simultaneously to both the secondary multicast groups and the primary users, the signals intended for secondary users might interfere the reception at the primary users' receivers. Upon defining $\check{P}_{m, k}$ as the power spent for transmitting to secondary user $m$ in group $g$ on subcarrier $k$ and denoting $T_{s}$ the OFDM symbol duration, the power spectral density (PSD)

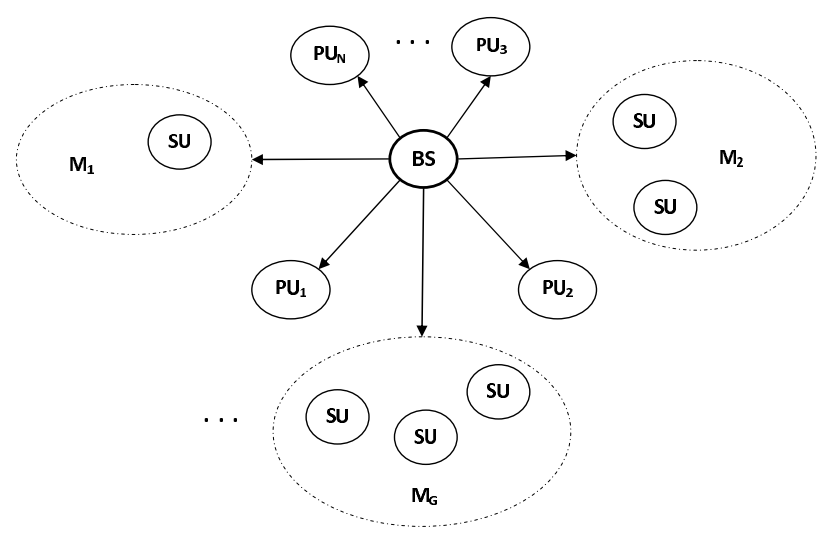

Fig. 1. Downlink transmission from BS to primary users (PU) and to multicast groups of secondary users (SU)

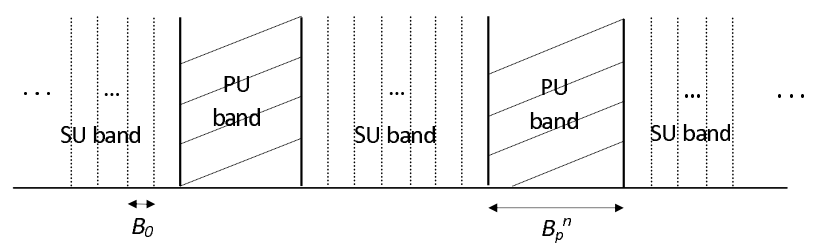

Fig. 2. Distribution of available spectrum to primary and secondary users

of the subcarrier- $k$ signal can be modelled as

$$
\Phi_{k}(f)=\check{P}_{m, k} T_{s}\left(\frac{\sin \pi f T_{s}}{\pi f T_{s}}\right)^{2}, m \in M_{g} .
$$

Then, the interference caused by this signal onto primary user $n$ can be expressed as [3]

$$
\begin{aligned}
I_{k}^{n} & =\left|g_{n}\right|^{2} \int_{d_{k}^{n}-B_{P}^{n} / 2}^{d_{k}^{n}+B_{P}^{n} / 2} \Phi_{k}(f) \mathrm{d} f \\
& =\check{P}_{m, k}\left\{\left|g_{n}\right|^{2} T_{s} \int_{d_{k}^{n}-B_{P}^{n} / 2}^{d_{k}^{n}+B_{P}^{n} / 2}\left(\frac{\sin \pi f T_{s}}{\pi f T_{s}}\right)^{2} \mathrm{~d} f\right\} \\
& =\check{P}_{m, k} \check{I}_{k}^{n},
\end{aligned}
$$

where $d_{k}^{n}=\left|f_{k}-f_{n}\right|$ represents the spectral distance between subcarrier $k$ and the center frequency of primary user $n$, and $g_{n}$ denotes the channel gain from BS to primary user $n$. Clearly, interference $I_{k}^{n}$ depends on both $d_{k}^{n}$ and $\check{P}_{m, k}$.

Due to the coexistence of primary users and multicast groups of secondary users, there is also interference induced by the signals, which are destined to primary users, onto secondary users' frequency bands. Let $h_{m, k}$ be the channel gain from BS to secondary user $m$ in group $g$ on subcarrier $k$, and $\Phi_{P U}^{n}\left(e^{j w}\right)$ be the PSD of the signal transmitted from BS to primary user $n$. Then, the interference power caused by this signal onto secondary user $m \in M_{g}$ on subcarrier $k$ can be modelled as [3]

$$
J_{m, k}^{n}\left(d_{k}^{n}\right)=\left|h_{m, k}\right|^{2} \int_{d_{k}^{n}-B_{s} / 2}^{d_{k}^{n}+B_{s} / 2} \mathbf{E}\left\{I_{K}(w)\right\} \mathrm{d} w,
$$

where $\mathbf{E}\left\{I_{K}(w)\right\}=\frac{1}{2 \pi K} \int_{-\pi}^{\pi} \Phi_{P U}^{n}\left(e^{j w}\right)\left(\frac{\sin (w-\phi) K / 2}{\sin (w-\phi) / 2}\right)^{2} \mathrm{~d} \phi$ is the PSD of primary user $n$ 's signal after $K$-FFT processing. 
In this work, the resource allocation is accomplished in a centralized manner at the BS, which has the perfect channel state information (CSI) of all the users (both primary and secondary) in the systems via feedbacks from the users through dedicated channels. The BS is able to determine the maximum rate at which an individual user can reliably receive data, as well as the corresponding subcarrier over which the data shall be transmitted on. It can be shown that the channel-tointerference-plus-noise ratio (CINR) of secondary user $m \in$ $M_{g}$ on subcarrier $k$ is

$$
\alpha_{m, k}=\frac{\left|h_{m, k}\right|^{2}}{\Gamma\left(N_{0} B_{s}+\sum_{n=1}^{N} J_{m, k}^{n}\right)},
$$

where $N_{0}$ is the one-sided PSD of additive white Gaussian noise (AWGN), and $\Gamma$ representing the signal-to-noise ratio (SNR) gap to capacity is a function of desired Bit-ErrorRate (BER), coding gain and noise margin. The maximum attainable rate of secondary user $m \in M_{g}$ on subcarrier $k$ can then be expressed as

$$
r_{m, k}=\frac{B_{s}}{B} \log _{2}\left(1+\alpha_{m, k} \check{P}_{m, k}\right) .
$$

In multicast systems, while all users within a group receive the same rate from the BS, the main difficulty arises from the mismatch data rates attainable by individual users in the group. If the BS transmits rate higher than the maximum rate that a user can handle, that user is not able to decode any of the transmitted data at all. A feasible, but yet simple scheme adopted in this paper is to transmit at the lowest rate of all the users within a group, which is determined by the user with the smallest CINR. On subcarrier $k$, let $\gamma_{g, k}=\min _{m \in M_{g}} \alpha_{m, k}$ be the equivalent CINR of group $g$, then the maximum rate at which all secondary users of $g$ are able to decode the transmitted data is

$$
\check{r}_{g, k}=\frac{B_{s}}{B} \log _{2}\left(1+\gamma_{g, k} P_{g, k}\right),
$$

where $P_{g, k}$ denotes power allocated to group $g$ on subcarrier $k$. As all the secondary users in a group receive the same rate, the aggregate rate transmitted to group $g$ on subcarrier $k$ is

$$
R_{g, m}=\sum_{m \in M_{g}} \check{r}_{g, k}=\left|M_{g}\right| \check{r}_{g, k} .
$$

The goal of this paper is to devise a joint subcarrier assignment and power allocation policy that maximizes the weighted sum rate of all multicast groups of secondary users, while satisfying constraints on the tolerable interference level of each individual primary user. Specifically, the design problem can be formulated as follows:

$$
\begin{array}{cl}
\max _{\left\{P_{g, k}\right\}} & \sum_{g=1}^{G} \sum_{k=1}^{K} \frac{w_{g}\left|M_{g}\right|}{K} \log _{2}\left(1+\gamma_{g, k} P_{g, k}\right) \\
\text { s.t. } & \sum_{g=1}^{G} \sum_{k=1}^{K} P_{g, k} \check{I}_{k}^{n} \leq I_{\mathrm{th}}^{n} ; n=1, \cdots, N, \\
& P_{g, k} \geq 0 ; g=1, \cdots, G, k=1, \cdots, K, \\
& P_{g, k} P_{g^{\prime}, k}=0 ; \forall g^{\prime} \neq g,
\end{array}
$$

In this formulation, $w_{g} \geq 0$ (where $\sum_{g=1}^{G} w_{g}=1$ ) is the weight denoting the priority designated to group $g$. Constraint (9) expresses the tolerable interference level at the receiver of primary user $n$, with $I_{\mathrm{th}}^{n}$ representing the interference temperature threshold. Constraints (10)-(11) enforce a disjoint subchannel assignment in OFDMA systems, i.e., one subcarrier is allowed to be assigned to at most one group at a time.

It is note-worthy that the optimization problem (8)-(11) is NP-hard as it requires the allocation of an optimal set of subcarriers to each multicast group of secondary users. Computational complexity of this combinatorial problem increases exponentially with the number of subcarriers $K$, which can be very large in practice, and thus the task of determining its optimal solution within a given time is very difficult. In the following sections, we will first introduce the dual optimization method for non-convex multicarrier resource allocation. Then, we will show how the seemingly challenging problem (8)-(11) can be solved in the dual domain with virtually zero duality gap, and thereby global optimal solutions can be obtained in the limit as the number of subcarriers goes to infinity. Further, we will show that the complexity of the proposed dual scheme is only linear in the number of subcarriers, which represents a huge reduction in computational burden at the BS where the optimal solutions are required to be found within a given time period due to quick variations of wireless channels.

\section{Dual Optimization Of Non-CONVEX MulticARrier Resource Allocation}

Consider the problem of optimally allocating resources in a multicarrier system with $M$ users and $K$ subcarriers. The objective and constraints of the optimization problem consist of a number of individual functions, each corresponding to one of the $K$ subcarriers, and can be expressed as

$$
\begin{aligned}
\max _{\left\{\mathbf{x}_{k}\right\} \in \mathcal{R}^{M}} & \sum_{k=1}^{K} f_{k}\left(\mathbf{x}_{k}\right) \\
\text { s.t. } & \sum_{k=1}^{K} \mathbf{h}_{k}\left(\mathbf{x}_{k}\right) \leq \mathbf{P},
\end{aligned}
$$

where $f_{k}(\cdot)$ are $\mathcal{R}^{M} \rightarrow \mathcal{R}$ (not necessarily concave) functions, $\mathbf{h}_{k}(\cdot)$ are $\mathcal{R}^{M} \rightarrow \mathcal{R}^{N}$ (not necessarily convex) functions, and constant $\mathbf{P}$ denotes the $N$-vector of constraints.

The idea of dual optimization is to solve (12) by first forming its Lagrangian dual, which is defined as

$$
\mathcal{L}\left(\left\{\mathbf{x}_{k}\right\}, \boldsymbol{\lambda}\right)=\sum_{k=1}^{K} f_{k}\left(\mathbf{x}_{k}\right)-\boldsymbol{\lambda}^{T}\left(\sum_{k=1}^{K} \mathbf{h}_{k}\left(\mathbf{x}_{k}\right)-\mathbf{P}\right),
$$

where $\boldsymbol{\lambda}=\left[\lambda_{1}, \cdots, \lambda_{N}\right]^{T} \succeq \mathbf{0}$ is a vector of Lagrange dual variables. Then, upon defining the dual objective $D(\boldsymbol{\lambda})=$ $\max _{\left\{\mathbf{x}_{k}\right\}} \mathcal{L}\left(\left\{\mathbf{x}_{k}\right\}, \boldsymbol{\lambda}\right)$, the dual optimization problem of (12) becomes

$$
\begin{array}{ll}
\min _{\boldsymbol{\lambda}} & D(\boldsymbol{\lambda}) \\
\text { s.t. } & \boldsymbol{\lambda} \succeq \mathbf{0}
\end{array}
$$


Let $f^{*}$ and $D^{*}$ denote the primal and the dual optimal value, respectively. From duality theory, the duality gap $d=D^{*}-f^{*}$ has been shown to be non-negative. When $f_{k}\left(\mathbf{x}_{k}\right)$ 's are concave and $\mathbf{h}_{k}\left(\mathbf{x}_{k}\right)$ 's are convex, strong duality is guaranteed, i.e. duality gap becomes zero, and the primal and the dual problems have the same optimal value. Zero duality gap implies that global optimum can be obtained in the dual domain via Lagrangian decomposition. In general, however, the gap between dual and primal optimum is not always zero.

Significantly enough, $[7, \mathrm{Sec} . \mathrm{III}]$ has shown that even if the multicarrier optimization problem (12) is non-convex, it has a zero duality gap whenever a so-called frequency-sharing condition is satisfied, which is always true in the limit as the number of subcarriers $K$ goes to infinity. This important result allows the original challenging non-convex problem to be efficiently solved in the dual domain with virtually negligible duality gap for a realistically large number of subcarriers.

\section{JoInt Optimal SUbCARRIER AND POWER Allocation Via DuALity}

It can be observed that the particular structure of problem (8)-(11) satisfies the frequency-sharing condition, and hence its global optimum can be obtained in the dual domain by an iterative method, at a significantly reduced computational complexity [7]. Specifically, for a fixed Lagrange dual variable set, it is possible to first decompose (8)-(11) by Lagrangian into several unconstrained per-tone power allocation subproblems, each of which can be solved by water-filling. Once the optimal distribution of powers is found for all subcarriers, the Lagrange dual variables are updated by a subgradient-based method. The procedure is repeated until convergence, and the joint optimal solution of subcarrier and power allocation obtained in the dual domain becomes that of the primal problem (8)-(11) as the number of subcarriers goes to infinity.

\section{A. The Proposed Dual Scheme}

The exclusive channel assignment constraint (10)-(11) can be expressed as $P_{g, k} \in \mathcal{S}$, where domain $\mathcal{S}$ is defined as $\mathcal{S}=\left\{P_{g, k} \geq 0 ; g=1, \cdots, G, k=1, \cdots, K \mid P_{g, k} P_{g^{\prime}, k}=\right.$ $\left.0, \forall g^{\prime} \neq g\right\}$. Over the domain $\mathcal{S}$, the Lagrangian of problem (8)-(9) can be written as

$$
\begin{aligned}
\mathcal{L}\left(\left\{P_{g, k}\right\}, \boldsymbol{\lambda}\right)= & \sum_{g=1}^{G} \sum_{k=1}^{K} \frac{w_{g}\left|M_{g}\right|}{K} \log _{2}\left(1+\gamma_{g, k} P_{g, k}\right) \\
& -\sum_{n=1}^{N} \lambda_{n}\left(\sum_{g=1}^{G} \sum_{k=1}^{K} P_{g, k} \check{I}_{k}^{n}-I_{\mathrm{th}}^{n}\right),
\end{aligned}
$$

where $\boldsymbol{\lambda}=\left[\lambda_{1}, \cdots, \lambda_{N}\right]^{T} \succeq \mathbf{0}$ is the vector of dual variables.

Now, thanks to the disjoint subchannel constraint in OFDMA, it is possible to decompose the Lagrange dual function of (15) into $K$ independent optimization problems, one for each subcarrier $k$, as follows:

$$
\begin{aligned}
D(\boldsymbol{\lambda}) & =\max _{\left\{P_{g, k}\right\} \in \mathcal{S}} \mathcal{L}\left(\left\{P_{g, k}\right\}, \boldsymbol{\lambda}\right) \\
& =\sum_{k=1}^{K} D_{k}(\boldsymbol{\lambda})+\sum_{n=1}^{N} \lambda_{n} I_{\mathrm{th}}^{n},
\end{aligned}
$$

where the per-subcarrier problem can be expressed as

$$
\begin{array}{r}
D_{k}(\boldsymbol{\lambda})=\max _{\left\{P_{g, k}\right\} \in \mathcal{S}} \sum_{g=1}^{G}\left\{\frac{w_{g}\left|M_{g}\right|}{K} \log _{2}\left(1+\gamma_{g, k} P_{g, k}\right)\right. \\
\left.-\left(\sum_{n=1}^{N} \lambda_{n} \check{I}_{k}^{n}\right) P_{g, k}\right\},
\end{array}
$$

for $k=1, \cdots, K$.

For each subcarrier $k$, there is at most one $P_{g, k}>0$ for all $g=1, \cdots, G$. Assume that multicast group $g$ is active on subcarrier $k$. For a fixed $\boldsymbol{\lambda}$, the objective of the maximization in (17) is a concave function of $P_{g, k}$. Hence, from the KKT conditions, the optimal power allocation can be derived as

$$
P_{g, k}^{*}=\left(\frac{1}{\gamma_{0, k}}-\frac{1}{\gamma_{g, k}}\right)^{+},
$$

where $x^{+}=\max (x, 0)$. Apparently, this is a form of waterfilling where the water level is indeed

$$
\gamma_{0, k}=\frac{K\left(\sum_{n=1}^{N} \lambda_{n} \check{I}_{k}^{n}\right) \log 2}{w_{g}\left|M_{g}\right|} .
$$

Then, by searching over all $G$ possible group assignments for subcarrier $k$, the optimal value of (17) can be obtained as

$$
\begin{aligned}
D_{k}^{*}(\boldsymbol{\lambda})= & \max _{g}\left\{\frac{w_{g}\left|M_{g}\right|}{K} \log _{2}\left[1+\gamma_{g, k}\left(\frac{1}{\gamma_{0, k}}-\frac{1}{\gamma_{g, k}}\right)^{+}\right]\right. \\
& \left.-\left(\sum_{n=1}^{N} \lambda_{n} \check{I}_{k}^{n}-I_{\mathrm{th}}^{n}\right)\left(\frac{1}{\gamma_{0, k}}-\frac{1}{\gamma_{g, k}}\right)^{+}\right\}
\end{aligned}
$$

for $k=1, \cdots, K$. To this end, the resource allocation on subcarrier $k$ is simply $P_{g^{*}, k}=P_{g^{*}, k}^{*}$ and $P_{g, k}=0$ for all $g \neq g^{*}$, where $g^{*}$ represents the group being allocated the subcarrier $k$.

Once (20) is solved for all subcarriers $k$ 's, the overall Lagrange dual function $D(\boldsymbol{\lambda})$ in (16) can be evaluated for the fixed $\boldsymbol{\lambda}$. Finally, it remains to finding $\boldsymbol{\lambda}^{*} \succeq \mathbf{0}$ that minimizes $D(\boldsymbol{\lambda})$. This can be efficiently done by iteratively updating $\boldsymbol{\lambda}$ utilizing a subgradient-based method until convergence of $\boldsymbol{\lambda}$. At that point, the sum interference, induced by transmission from the BS to all multicast groups, to each primary user's frequency band also converges, and (positive) optimal powers have been distributed to the eligible multicast groups.

The update of $\boldsymbol{\lambda}$ may be performed as follows:

$$
\lambda_{n}^{(t+1)}=\left(\lambda_{n}^{(t)}-\delta^{(t)}\left(I_{\mathrm{th}}^{n}-\sum_{g=1}^{G} \sum_{k=1}^{K} P_{g, k} \check{I}_{k}^{n}\right)\right)^{+},
$$

for $n=1, \cdots, N$, where $\delta^{(t)}>0$ is a sequence of scalar step size. This subgradient update is guaranteed to converge to the 
optimal $\lambda^{*}$ as long as $\delta^{(t)}$ is chosen to be sufficiently small. A popular choice is that $\delta^{(t)}$ is square summable but not absolute summable, for instance, $\delta^{(t)}=\frac{\beta}{t}$, for some constant $\beta>0$.

TABLE I

JOINT SUBCARRIER AND POWER ALLOCATION BY DUAL METHOD

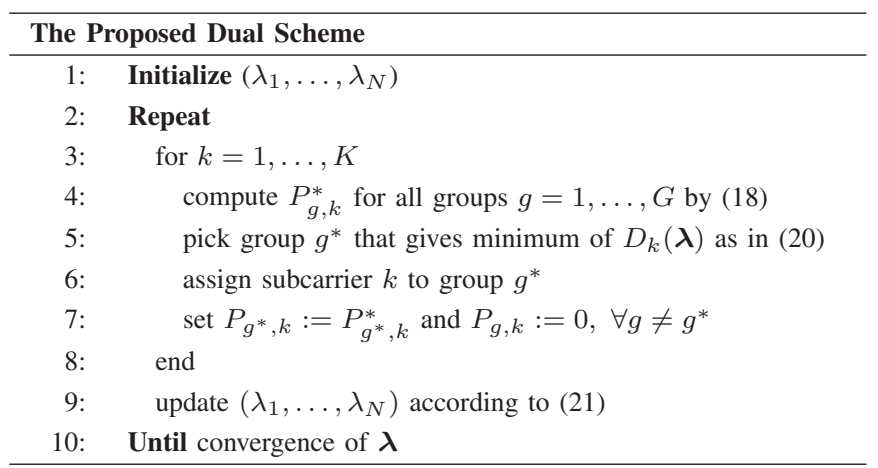

The overall proposed dual scheme is summarized in Table I. It is important to point out that although the sum interference power caused by all multicast groups to each of the primary users do not always converge to the interference threshold $I_{\mathrm{th}}^{n}$ in the proposed scheme, the gap $\sum_{g=1}^{G} \sum_{k=1}^{K} P_{g, k} \check{I}_{k}^{n}-I_{\mathrm{th}}^{n}$ vanishes quickly as the number of subcarriers goes to infinity, hence the duality gap becomes zero. In practice, OFDM systems employ very large number of subcarriers (e.g., as many as 4096), and thus the optimal solution, obtained in the dual domain by the proposed scheme, essentially becomes a global optimum of the primal problem (8)-(11), with a negligible duality gap.

\section{B. Complexity Analysis}

For a fixed $\lambda$, solving problem (16) requires $\mathcal{O}(K G)$ executions. An ellipsoid method used to update $\lambda$ converges in $\mathcal{O}\left(N^{2}\right)$ iterations. Therefore, the total complexity of the proposed dual scheme is $\mathcal{O}\left(K G N^{2}\right)$, which is only linear in the number of subcarriers. Since the number of subcarriers is often large in practical settings, there is a huge reduction in computational complexity by the proposed dual scheme. This is a very desirable feature of algorithms designed for wireless communication systems where optimal solutions are often required to be found within a very short time due to the dynamics of wireless channels.

\section{Effects of Adjacent Subcarrier Nulling}

The study in [3] proposes the method of dynamically deactivating subcarriers as a countermeasure to reduce the amount of interference from secondary to primary bands. Essentially, the suggested approach provides flexible guard bands between primary and secondary users by nulling subcarriers which are adjacent to the primary users' bands. However, this benefit comes at the cost of sacrificing bandwidth and hence throughput of secondary users. It is therefore critical to balance the contradicting requirements of reducing interference and achieving highest possible throughput of secondary users.
The computational complexity needed to find the optimal solutions in the proposed dual scheme can be further reduced by applying the adjacent subcarrier nulling method since the number of subcarriers $K$ decreases. As well, more power can be distributed into the far-away subcarriers for a given interference threshold $I_{\mathrm{th}}^{n}$. However, nulling adjacent subcarriers also reduces the available degree of freedom, which is the number of available subcarriers for possible transmission from the BS to secondary users, and leads to a decrease in the total sum rate achieved by all multicast groups. Therefore, only the deactivation of first and second adjacent subcarriers on each side of primary users' bands are recommended, as additional nulling merely provides marginal advantages.

\section{NUMERICAL EXAMPLES}

Consider a wireless system in which the BS communicates with $N=2$ primary users. The frequency reserved for transmission to each primary user is predetermined in the available spectrum. All the signals transmitted to the primary users are assumed to be elliptically filtered white noise with equal amplitude $P_{P U}=1$. As the primary users do not utilize the whole available spectrum, the BS is also allowed to simultaneously transmit to $G=2$ multicast groups each consists of $\left|M_{1}\right|=\left|M_{2}\right|=3$ secondary users, over $K=24$ OFDM subcarriers in the unused frequency bands, which are located on the sides of those already occupied bands. To have a meaningful interpretation of the results, 100 sets of independent channel gains $\left\{g_{n}\right\}$ and $\left\{h_{m, k}\right\}$ are randomly generated according to the Rayleigh distribution in the computation of attainable sum rates. For simplicity, the average channel gains, the noise power of each subcarrier, the OFDM symbol duration, and the individual subcarrier bandwidth are all normalized to 1 . It is further assumed that perfect coding is employed, thus $\Gamma=1$. Since all the spectral distances $d_{k}^{n}$ can be determined, it is possible to compute the interferences $\check{I}_{k}^{n}$, $J_{m, k}^{n}$, and the CINR of individual secondary user $\alpha_{m, k}$. Then, the equivalent CINR of group $M_{g}$ on subcarrier $k$ is simply $\gamma_{g, k}=\min _{m \in M_{g}} \alpha_{m, k}$. Assume that $M_{1}$ is closer to the BS and causes a pathloss difference of $2.5 \mathrm{~dB}$ between $M_{1}$ and $M_{2}$. The interference threshold at individual primary user's receiver is assumed to be equal $I_{\mathrm{th}}^{1}=I_{\mathrm{th}}^{2}=I_{\mathrm{th}}$, and both groups also have equal priority, i.e, $w_{1}=w_{2}=0.5$.

Fig. 3 plots the total sum rate as well as individual group rates obtained by the proposed scheme, with and without nulling adjacent subcarriers on each side of the primary users' bands. It should be noted that only deactivating 1 and 2 adjacent subcarriers are shown since additional deactivation just brings minor benefits. For a random channel realization at interference threshold $I_{\mathrm{th}}=.10$, Fig. 4 and Fig. 5 illustrate the convergence of the sum interference introduced to each primary user (PU) and that of the dual variables, respectively. Table II shows the gap between the required interference threshold and the actual total interference at individual PU's band when subcarrier nulling is not implemented.

It can be seen from Fig. 3 that the multicast group with better channel condition (i.e., $M_{1}$ ) achieves higher rate as it 


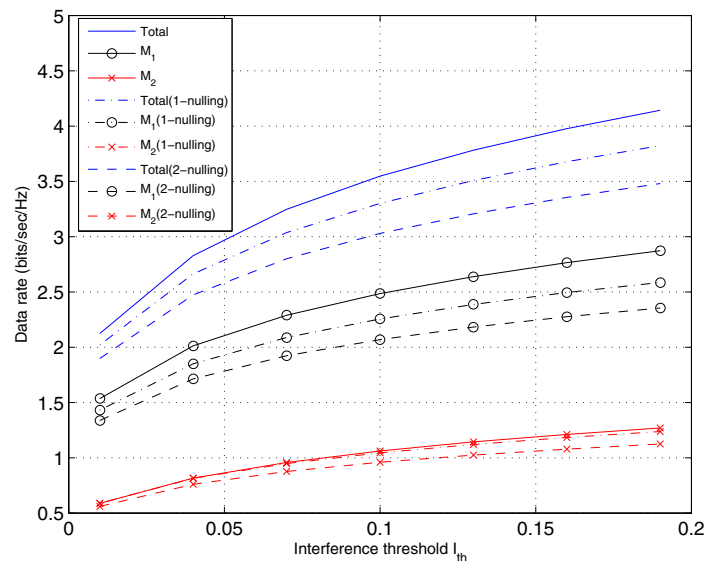

Fig. 3. Attained data rates by the proposed dual scheme

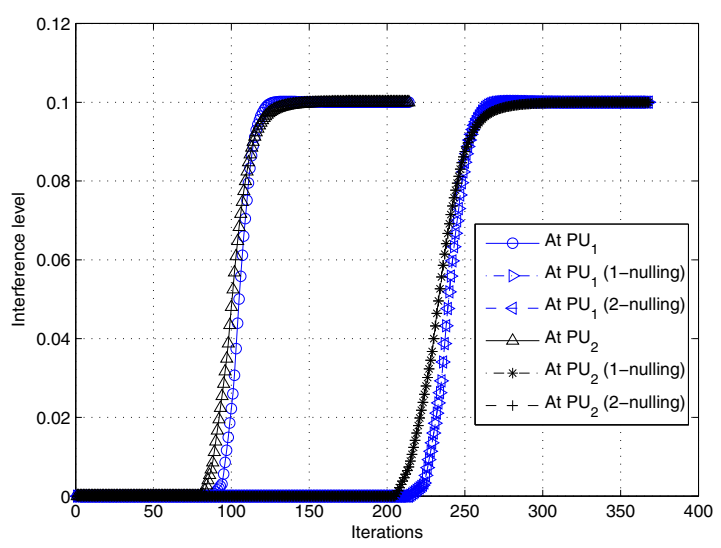

Fig. 4. Convergence of sum interference introduced to primary users

is given more subchannels in the unused spectrum. Moreover, the attainable sum rates in the 1 -nulling and 2 -nulling cases actually decrease, since the adjacent subcarriers are assigned zero power even when their respective channel conditions are very good. Additional nulling is thus expected to cause further reduction in the achieved sum rates. Figs. 4 and 5 confirm the convergence of the proposed dual scheme after only few hundreds of iterations. Also provided in Table II is the gap between the interference threshold and the total interference introduced to each primary user's band, which is shown to be negligible even when only $K=24$ subcarriers are employed. This illustrates that the solution obtained by the proposed dual method is very close to the primal global optimum. It is therefore reasonable to expect that this gap tends to zero when the number of subcarriers becomes much larger, which is often the case in practice.

\section{CONCLUSION}

In this paper, we have proposed a dual scheme for optimal allocation of subcarriers and power to maximize the total sum rate of a secondary network employing OFDMA, subject

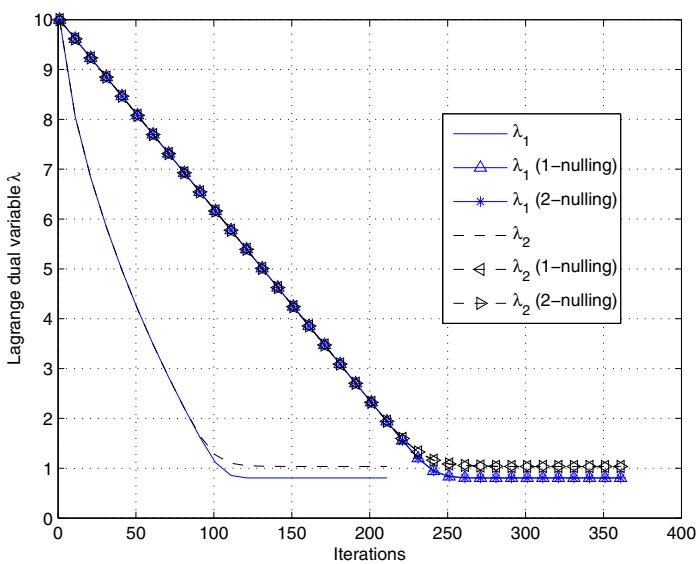

Fig. 5. Convergence of Lagrange dual variables $\boldsymbol{\lambda}$

TABLE II

GAPS BETWEEN SUM INTERFERENCE AND INTERFERENCE THRESHOLD $\left(\times 10^{-5}\right)$

\begin{tabular}{|l|r|r|r|r|r|r|r|}
\hline Threshold & .01 & .04 & .07 & .10 & .13 & .16 & .19 \\
\hline At $\mathbf{P U}_{1}$ & .5757 & .0643 & .0748 & .0623 & .0539 & .0546 & .0567 \\
\hline At $\mathbf{P U}_{2}$ & .9330 & .3971 & .2384 & .1910 & .1486 & .1142 & .0836 \\
\hline
\end{tabular}

to tolerable interference at the primary users. The proposed method can deal with both unicast and multicast transmissions, and its complexity is linear in the number of subcarriers. The effects of adjacent subcarrier nulling have also been investigated, and the numerical results have confirmed the potential benefits of the proposed solution.

\section{ACKNOWLEDGEMENT}

This work is supported in part by Alberta Ingenuity Student Scholarship and $i$ CORE Graduate Student Scholarship.

\section{REFERENCES}

[1] J. Mitola and G. Q. Maguire, "Cognitive radio: Making software radios more personal," IEEE Personal Commun. Mag., vol. 6, no. 4, pp. 13-18, Aug. 1999.

[2] T. Weiss and F. Jondral, "Spectrum pooling: an innovative strategy for the enhancement of spectrum efficiency," IEEE Commun. Mag., vol. 42, no. 3, pp. S8-14, Mar. 2004.

[3] T. Weiss, J. Hillenbrand, A. Krohn, and F. Jondral, "Mutual interference in OFDM-based spectrum pooling systems," in Proc. IEEE Vehicular Technology Conf. (VTC), vol. 4, May 2004, pp. 1873-1877.

[4] G. Bansal, M. Hossain, and V. Bhargava, "Adaptive power loading for OFDM-based cognitive radio systems," in Proc. IEEE Int. Conf. Communications (ICC), Jun. 2007, pp. 5137-5142.

[5] T. Qin and C. Leung, "Fair adaptive resource allocation for multiuser OFDM cognitive radio systems," in Proc. Second Int. Conf. Communications and Networking in China (CHINACOM), Aug. 2007, pp. 115-119.

[6] P. Cheng, Z. Zhang, H. Huang, and P. Qiu, "A distributed algorithm for optimal resource allocation in cognitive OFDMA systems," in Proc. IEEE Int. Conf. Communications (ICC), May 2008, pp. 4718-4723.

[7] W. Yu and R. Lui, "Dual methods for nonconvex spectrum optimization of multicarrier systems," IEEE Trans. Commun., vol. 54, no. 7, pp. 1310 1322, Jul. 2006.

[8] U. Varshney, "Multicast over wireless networks," Commun. of the ACM, vol. 45, pp. 31-37, Dec. 2002.

[9] K. Seong, M. Mohseni, and J. M. Cioffi, "Optimal resource allocation for OFDMA downlink systems," in Proc. IEEE Int. Symposium on Inform. Theory, Jul. 2006, pp. 1394-1398. 